\title{
Stress Relaxation in Entangled Polymer Melts
}

\author{
Ji-Xuan Hou, ${ }^{1}$ Carsten Svaneborg, ${ }^{2}$ Ralf Everaers, ${ }^{1}$ and Gary S. Grest ${ }^{3}$ \\ ${ }^{1}$ Laboratoire de Physique and Centre Blaise Pascal of the École \\ Normale Supérieure de Lyon, Université de Lyon, CNRS UMR 5672, \\ 46, allée d'Italie, 69364 Lyon cedex 0\%, France \\ ${ }^{2}$ Department of Chemistry and Interdisciplinary Nanoscience Center (iNANO), \\ University of Aarhus, Langelandsgade 140, DK-8000 Aarhus C, Denmark \\ ${ }^{3}$ Sandia National Laboratories, Albuquerque, NM 87185, USA
}

\begin{abstract}
We present an extensive set of simulation results for the stress relaxation in equilibrium and stepstrained bead-spring polymer melts. The data allow us to explore the chain dynamics and the shear relaxation modulus, $G(t)$, into the plateau regime for chains with $Z=40$ entanglements and into the terminal relaxation regime for $Z=10$. Using the known (Rouse) mobility of unentangled chains and the melt entanglement length determined via the primitive path analysis of the microscopic topological state of our systems, we have performed parameter-free tests of several different tube models. We find excellent agreement for the Likhtman-McLeish theory using the double reptation approximation for constraint release, if we remove the contribution of high-frequency modes to contour length fluctuations of the primitive chain.
\end{abstract}

PACS numbers: 83.80.Sg (Polymer melts), 83.10.Rs (MD simulation rheology), 61.25.he (liquid structure polymer melt)

High molecular weight polymeric liquids display remarkable viscoelastic properties [1, 2]. Contrary to glassy systems, their macroscopic relaxation times are not due to slow dynamics on the monomer scale, but arise from the chain connectivity and the restriction that the backbones of polymer chains cannot cross while undergoing Brownian motion. Modern theories of polymer dynamics [3, 4] describe the universal aspects of the viscoelastic behavior based on the idea that molecular entanglements confine individual polymers to tube-like regions in space $[5,[6]$. Forty years of research have led to a complex relaxation scenario based on a combination of local Rouse dynamics, reptation, contour length fluctuations, and constraint release [4]. The development and validation of a quantitative, microscopic theory crucially depends on the availability of experimental and simulation data for model systems.

Entangled polymers are studied experimentally using rheology [1, 2, 7], dielectric spectroscopy [8], small-angle neutron scattering [9, 10], and nuclear magnetic resonance [11, 12]. Computer simulations [13 16] offer some advantages in the preparation of well-defined model systems and the simultaneous access to macroscopic behavior and microscopic structure and dynamics. In particular, the recently developed primitive path analysis (PPA) 17 24 reveals the experimentally inaccessible mesoscopic structures and relaxation processes described by the tube model and allows parameter-free comparisons between theoretical predictions and data. However, the long relaxation times pose a particular challenge to computational techniques. Here we present simulation results for model polymer melts in equilibrium and after a rapid, volume-conserving uni-axial elongation, where we have been able to follow the full relaxation dynamics deep into the entangled regime. The data allow us to perform the first parameter-free test of the predictions of tube models for dynamical properties, to pinpoint a problem in the current theoretical description, and to validate a suitable modification.

Our numerical results are based on extensive molecular dynamics (MD) simulations of bead-spring polymer melts [13]. Each chain is represented as a sequence of beads connected by finite-extensible, non-linear (FENE) springs and interacting via the repulsive part of the Lennard-Jones 12-6 potential (LJ). The energy scale is set by the strength of the LJ interaction, $\epsilon$, while the distance scale is set by the monomer size, $\sigma$. The basic unit of time is $\tau=\sigma(m / \epsilon)^{1 / 2}$, where $m$ is the mass of each monomer. The equations of motion are integrated using the LAMMPS MD simulation package 25] with a velocity Verlet algorithm and a time step $\delta t=0.012 \tau$. The temperature, $T=\epsilon / k_{B}$, was kept constant by weakly coupling the motion of each bead to a heat bath with a local friction $\Gamma=0.5 \tau^{-1}$.

We have studied seven entangled polymer melts of $M$ chains of $N$ beads with $M \times N=5000 \times 50,2500 \times 100$, $400 \times 175,200 \times 350,200 \times 700,400 \times 1000$, and $320 \times 3500$ each at a monomer density $\rho=0.85 \sigma^{-3}$. Using the most refined PPA estimate of the rheological entanglement length for this model of $N_{e}=85 \pm 7$ [36], the investigated systems span the range from unentangled $\left(Z=N / N_{e} \approx\right.$ $0.6)$ to highly entangled $\left(Z=N / N_{e} \approx 41\right)$. The Rouse time was previously determined as $\tau_{R}=1.5 \tau N^{2}[13]$, entangements effects become relevant around $\tau_{e}=\tau_{R}\left(N_{e}\right)$, and the maximal relaxation times of entangled systems are expected to be on the order of $\tau_{d}^{0}=\tau_{e} Z^{3}=\tau_{R} Z$.

The melts were generated and equilibrated following the procedure outlined in Auhl et al. [16]. Technically, the largest challenge is the reliable extraction of the macroscopic, viscoelastic behavior [26, 27]. Data were 


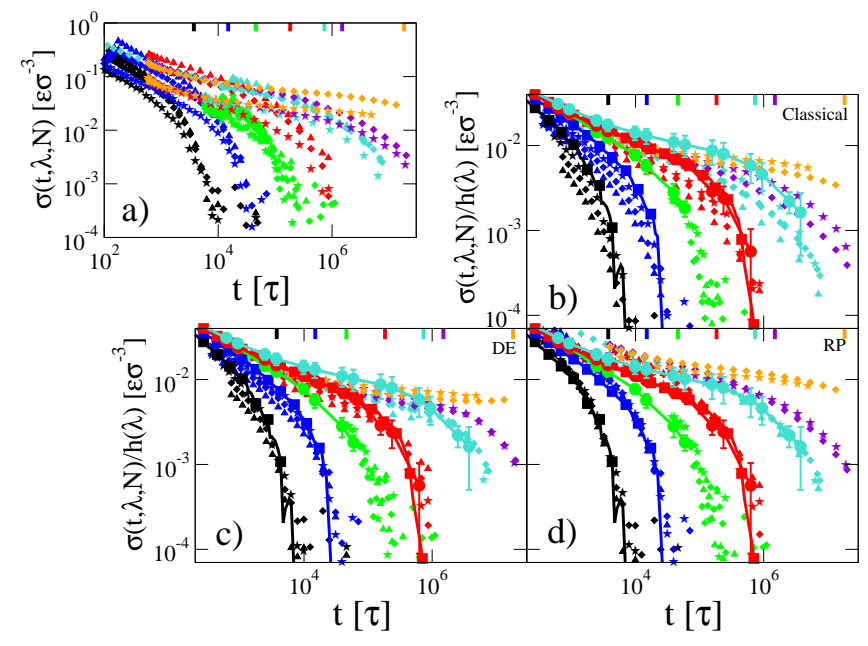

FIG. 1: (color online) (a) Normal tensions, $\sigma(\lambda, t)$ for stepstrained melts $N=50$ (black), 100 (blue), 175 (green), 350 (red), 700 (cyan), 1000 (violet), and 3500 (orange) and elongation $\lambda=2(\square), 3(\diamond)$, and $4(\triangle)$. (b-d) Green-Kubo shear relaxation moduli, $G(t)$, (this work: large solid $\bigcirc$ with solid line; Ref. [26]: no symbol, solid line) compared to extrapolations, $G(\lambda, t)=\sigma(\lambda, t) / h(\lambda, t)$, from the non-linear response using the (b) classical, (c) Doi-Edwards, (d) slip-tube damping function (same symbols as in (a)). Colored ticks indicate the Rouse time of the corresponding systems.

recorded in equilibrium as well as out of equilibrium after a step-strain. Strained melts were prepared by subjecting equilibrated initial conformations to rapid $\left(T_{\text {def }} \in\right.$ $[120 \tau, 36000 \tau])$, volume-conserving $\left(\lambda_{x} \lambda_{y} \lambda_{z} \equiv 1\right)$, elongational deformations with $\left(\lambda_{x}=\lambda, \lambda_{y}=\lambda_{z}=1 / \sqrt{\lambda}\right)$ for $\lambda$ ranging from 1.5 to 4.0 well outside the linear elastic regime. Deformations in this range are typical for many applications of polymeric systems and large enough to generate a measurable elastic response for the present system sizes. In the ideal case, strain should be introduced instantaneously. To check the dependance of our results on $T_{d e f}$, we have varied the deformation time in one case ( $N=700, \lambda=3.0$ ) by a factor of 200 . In the following, for deformed systems, $t=0$ is fixed to the middle of the deformation period, i.e. data are recorded for $t \geq T_{\text {def }} / 2$. To reduce finite- $T_{\text {def }}$ artifacts, we typically discard data from the initial $3 T_{\text {def }}$.

The longest simulations were run up to $2 \times 10^{9}$ time steps and sufficient to reach the plateau regime for our longest chains and to completely relax the others $\left(2 \times 10^{9} \times 0.012 \tau \approx \tau_{R}(N=4000) \approx \tau_{d}(Z=12.5)\right)$. The total numerical effort corresponds to about 5 million single core CPU hours. We recorded block-averages of the microscopic stress tensor $\sigma_{\alpha \beta}(t)=\left\langle\sum_{i j} F_{i j, \alpha} r_{i j, \beta}\right\rangle / V$ at intervals of $1.2 \tau$. The latter sum is over all pairs $i, j$ of interacting beads, $\alpha, \beta$ are Cartesian indices, and $F, r$ and $V$ denotes force, separation and volume, respectively. Furthermore, we stored melt conformations at intervals of $120 \tau$ for further analysis of the chain conformations.
Results for the relaxation of the normal tension $\sigma(t)=$ $\sigma_{x x}-\frac{1}{2}\left(\sigma_{y y}+\sigma_{z z}\right)$ are presented in Fig. 1. 1. We observe a clear non-Newtonian behavior with a stress relaxation extending over many orders of magnitude in time after the end of the deformation period of the sample. As expected, there is a strong increase of the terminal relaxation time with chain length and the gradual formation of an intermediate plateau in the stress relaxation for the longest chains studied. The maximal relaxation time is independent of the total deformation.

Figure 1(b-d) show comparisons between $\sigma(\lambda, t) / h(\lambda, t)$ for the step-strained melts to the linear shear relaxation moduli $G(t)$ obtained by Likhtman et al. 26] and ourselves via the Green-Kubo relation $G(t)=V\left\langle\sigma_{\alpha \beta}(t) \sigma_{\alpha \beta}(0)\right\rangle / k_{B} T$ with $\alpha \neq \beta$ the from stress fluctuations in unstrained, equilibrated melts. Available expressions for damping functions $h(\lambda)$ are refinements of the stress-strain relation $h(\lambda)=\lambda^{2}-\lambda^{-1}$ predicted by classical rubber elasticity theory (Fig. [b). The Doi and Edwards [28] damping function (Fig. 1F) includes the dynamics of a uniform chain retraction inside the stretched tube. The Rubinstein-Panyukov slip-tube damping function [29], $h(\lambda)=\left(\lambda^{2}-\lambda^{-1}\right) /\left(0.74 \lambda+0.61 \lambda^{-1 / 2}-0.35\right)$, accounts for non-affine tube deformations and the asymptotic chain length redistribution inside the tube (Fig. $1 \mathrm{l}$ ). For times $t<\tau_{R}(N)$, the presence of additional relaxation processes prevents a systematic extraction of $G(t)$ from normal tensions measured in the non-linear regime. Empirically, the time-independent slip-tube expression works surprisingly well. For times $t>\tau_{R}(N)$, the differences between the slip-tube and the Doi-Edwards damping functions are small. Both result in a satisfactory data collapse and good agreement with the Green-Kubo results, suggesting that they capture the non-linear effects at large strains with reasonable accuracy. The step-strain data included in Figs. 2 and 3 correspond to times $t>0.25 \tau_{R}(N)$.

In Figure $2 \mathrm{a}$ we show a comparison of the simulation results for $G(t)$ to the Rouse model predictions [3, 30] for unentangled systems. Our results confirm the expectation that the Rouse model quantitatively describes the chain length independent early-time stress relaxation with $G(t) \propto t^{-1 / 2}$ as well as terminal stress relaxation in systems where the chains are too short to be entangled. For longer chains, entanglements start to affect the behavior beyond a material-specific, characteristic time $\tau_{e} \approx 10^{4} \tau$ with a gradual formation a plateau in the stress relaxation reached by our longest chain systems with $Z=41$. For the terminal stress relaxation of systems with $Z=\mathcal{O}(10)$ we have reliable data extending about one order of magnitude below the plateau level. This is sufficient to allow for a meaningful comparison to current theories. In particular, we are not restricted to comparing the ability of different theories to fit the data. Rather, we can carry out absolute, parameter-free com- 


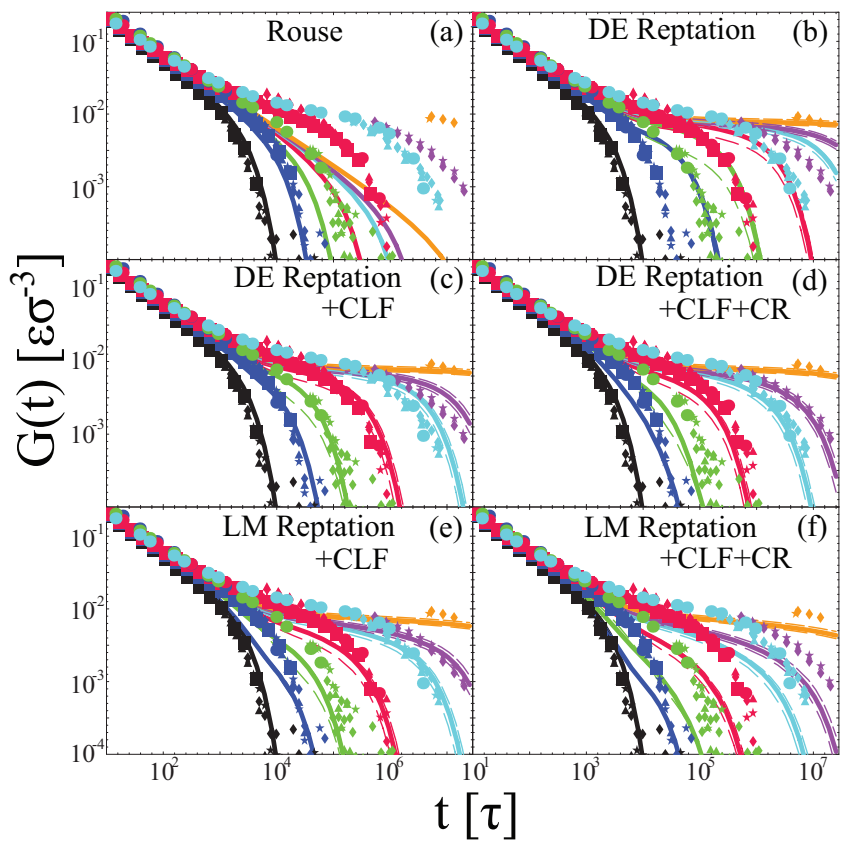

FIG. 2: (color online) Comparison of the measured relaxation moduli to the predictions of various theories. Symbols and colors for the simulation data as in Fig. 1 with Green-Kubo data shown as large solid $\bigcirc$ and $\square$. Theoretical predictions are shown as thick lines using the same color code. Thin lines indicate the uncertainty in the theoretical predictions due to the uncertainty of the PPA entanglement length $N_{e}=85 \pm 7$.

parisons using the result $N_{e}=85 \pm 7$ [24] of the primitive path analysis and the known Rouse friction of the model.

Likhtman and McLeish (LM) 31] assembled the effects of (i) early-time Rouse relaxation, (ii) tension equilibration along the contour of the primitive chains, (iii) reptation, (iv) contour length fluctuations, and (v) constraint release into a closed functional form,

$$
\begin{aligned}
G(t)= & \frac{\rho k_{B} T}{N} \frac{1}{5} \sum_{p=1}^{Z}\left(4 \mu(t) R(t)+e^{-t p^{2} / \tau_{R}}\right) \\
& +\frac{\rho k_{B} T}{N} \sum_{p=Z+1}^{N} e^{-2 t p^{2} / \tau_{R}}
\end{aligned}
$$

where $\mu(t)$ and $R(t)$ account for single- and multi-chain relaxation processes of the tube model. In their absence $(\mu(t)=R(t) \equiv 1)$, the formula describes a crossover from the early time Rouse relaxation $G(t) \propto t^{-1 / 2}$ to a plateau $G_{N}^{0}=\frac{4}{5} \frac{\rho k_{B} T}{N_{e}}$. The key quantity of the tube model is the single-chain memory function, $\mu(t)$, for the fraction of the primitive chain which has not escaped from its original tube after a time $t$. Comparisons to the data neglecting constraint release $(R(t) \equiv 1)$ are shown for the original Doi-Edwards model accounting only for reptation (Fig. 2b), Doi's [3] approximate inclusion of the effect of contour length fluctuations combining reptation dynamics with the maximal relaxation time from 31] (Fig. 2r),

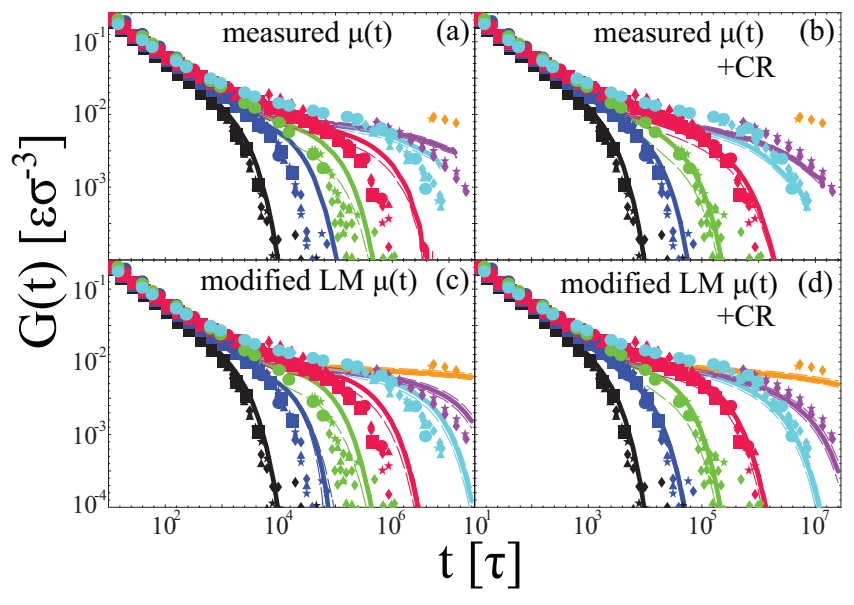

FIG. 3: (color online) Comparison of the measured relaxation moduli to Eq. (1) using (a,b) the independently measured autocorrelation function of the primitive chain end-to-end vectors to estimate of the tube memory function, $\mu(t) ;(\mathrm{c}, \mathrm{d})$ our proposition Eqs. (24) for removing high-frequency modes from the Likhtman-McLeish theory 31] of contour length fluctuations with $\alpha=1.7$. Symbols and colors as in Fig. 2

and the full LM theory [31] of contour length fluctuations and reptation (Fig. 2 2 ). For the comparisons in Figs. 2 and $\mathrm{f}$ we have included the effect of constraint release in the double reptation [32] approximation $R(t)=\mu(t)$.

The overall agreement between our data and the more advanced versions of the tube model is fairly good. Interestingly, the more sophisticated LM theory seems to work less well than Doi's approximation when combined with the LM estimate of the maximal relaxation time. Yet, from our rheological data alone, it is hard to clearly identify the relevance and the quality of the theoretical description of the various relaxation processes. For example, one might (as we believe, erroneously; see below) conclude, that the double reptation approximation strongly overestimates the contribution of constraint release to the stress relaxation (Figs. $2 \mathrm{f}$ and $\mathrm{f}$ ) or that constraint release is inefficient for $Z<4$ (Figs. 2 r and d). Obviously, fitting the various theories to the data would only obscure their shortcomings.

To draw definite conclusion on how to improve the theories, we discriminate between three possible sources of error: (i) the functional form of Eq. (11), (ii) the treatment of reptation and contour length fluctuations underlying the single-chain memory function $\mu(t)$, and (iii) the treatment of the multi-chain effect of constraint release via the double reptation approximation, $R(t)=\mu(t)$. For long chains and under the assumption that the escaped chain sections equilibrate completely, $\mu(t)$ equals the autocorrelation function of the chain end-to-end vectors [4], For shorter chains, it is more suitable to consider the endpoint motion of the primitive chains, defined as the average of the chain conformation over a period of $\tau_{e}[33$. This correlation function is easily accessible from our 
equilibrium simulations and is not affected by constraint release [4]. The comparison between the measured relaxation moduli and those predicted from Eq. (1) using the measured $\mu(t)$ together with $R(t) \equiv 1$ and $R(t)=\mu(t)$ is shown in Figs. 3 a and b respectively. For the full theory the agreement is excellent, supporting the utility of both the Likhtman-McLeish functional form of the shear relaxation modulus and of the double reptation approximation for constraint release.

The shortcomings of the LM description apparent in Fig. 2f and in the rheological study by Liu et al. 7] must thus be related to the central part of their theory, the estimation of the time dependence of $\mu(t)$ under the combined influence of reptation and contour length fluctuations. A possible explanation is a double-counting of the effect of short-wavelength $(p>Z)$ modes in the Rouse relaxation part of Eq. (1) and in $\mu(t)$. LM extrapolated $\mu(t)$ to the continuum limit, resulting in a decay on time scales $t<\tau_{e}$, where the motion of the primitive chain should be negligible. To correct for this, we have removed from the CLF part of $\mu(t)$ the contribution of modes with a relaxation time shorter than $\alpha^{4} \tau_{e}$ :

$$
\begin{aligned}
\mu(t)= & \left(1+\frac{1.22 \alpha}{Z}\right)\left(\frac{8 \widetilde{G}_{f}}{\pi^{2}} \sum_{p=1, \text { odd }}^{p^{*}} \frac{1}{p^{2}} \exp \left(-\frac{t p^{2}}{\tau_{d f}}\right)\right. \\
& \left.+\int_{\epsilon^{*}}^{1 /\left(\alpha^{4} \tau_{e}\right)} \frac{0.306}{Z \tau_{e}^{1 / 4} \epsilon^{5 / 4}} \exp (-\epsilon t) d \epsilon\right) \\
\frac{\tau_{d f}(Z)}{\tau_{R}}= & 3 Z\left(1-\frac{2 \times 1.69}{\sqrt{Z}}+\frac{4.17+1.22 \alpha}{Z}\right. \\
& \left.-\frac{1.55+2.69 \alpha}{Z^{3 / 2}}\right) \\
\widetilde{G}_{f}= & 1-\frac{1.69}{\sqrt{Z}}+\frac{2.0}{Z}-\frac{1.24-1.03 \alpha}{Z^{3 / 2}}
\end{aligned}
$$

where $p^{*}=\sqrt{Z / 10}$ and $\epsilon^{*}$ are defined as in the original LM theory, which is recovered in the $\alpha \equiv 0$ limit of the above expressions. Fig. $3 \mathrm{~d}$ shows that we obtain significantly improved agreement between theory and our data for values $\alpha=\mathcal{O}(1)$. Interestingly, this corresponds to a constant offset of $Z=Z_{L M}-$ $\int_{1 /\left(\alpha^{4} \tau_{e}\right)}^{\infty} \frac{0.306}{Z \tau_{e}^{1 / 4} \epsilon^{5 / 4}} \exp (-\epsilon t) d \epsilon=Z_{L M}-1.22 \alpha$. This view is in qualitative agreement with arguments put forward by van Ruymbeke et al. 34] to consider, within the original LM theory, chains with virtual extensions of length $N_{e}$ resulting in an increase the relaxation time of the outermost "real" chain segment to $\tau_{e}$.

To summarize, we have presented an extensive set of simulation results for the equilibrium and relaxation dynamics of entangled model polymer melts. In particular, we explored $G(t)$ into the plateau regime for chains with $Z=41$ and into the terminal relaxation regime for $Z \leq 10$ and compared our data to predictions of different versions of the tube model. These comparisons did not involve any free parameters, since the entangle- ment length was determined independently via a topological analysis [17, 24]. We find excellent agreement for the Liktman-McLeish theory using a corrected tube memory function and the double reptation approximation for constraint release, demonstrating that the primitive path analysis of the microscopic structure endows the tube model with predictive power for dynamical processes. The use of more elaborate schemes [35] for treating constraint release and predicting the function $R[\mu(t)]$ should lead to even better agreement.

We thank the New Mexico Computing Application Center NMCAC for generous allocation of computer time and A. Likhtman and S.K. Sukumaran for their GreenKubo data. CS acknowledges financial support from the Danish Natural Sciences Research Council through a Steno Research Assistant Professor fellowship. JXH is supported by the EC through the Marie Curie EST Eurosim Project No. MEST-CT-2005- 020491. RE acknowledges a chair of excellence grant from the Agence Nationale de Recherche (France). Sandia is a multiprogram laboratory operated by Sandia Corporation, a Lockheed Martin Company, for the U.S. Department of Energy under Contract No. DE-AC04-94AL85000.

[1] J. D. Ferry, Viscoelastic Properties of Polymers (Wiley, New York, 1980).

[2] R. B. Bird, R. C. Armstrong, and O. Hassager, Dynamics of Polymeric Liquids, vol. 1 (Wiley, New York, 1977).

[3] M. Doi and S. F. Edwards, The Theory of Polymer Dynamics (Clarendon, Oxford, 1986).

[4] T. C. B. McLeish, Adv. Phys. 5, 1379 (2002).

[5] S. F. Edwards, Proc. Phys. Soc. 92, 9 (1967).

[6] P. G. de Gennes, J. Chem. Phys. 55, 572 (1971).

[7] C.-Y. Liu, R. Keunings, and C. Bailly, Phys. Rev. Lett. 97, 246001 (2006).

[8] H. Watanabe, Prog. Polym. Sci. 24, 1253 (1999).

[9] B. Ewen and D. Richter, Adv. Pol. Sci. 134, 3 (1997).

[10] A. Wischnewski, M. Monkenbusch, L. Willner, D. Richter, A. E. Likhtman, T. C. B. McLeish, and B. Farago, Phys. Rev. Lett. 88, 058301 (2002).

[11] P. T. Callaghan and E. T. Samulski, Macromolecules 30, 113 (1997).

[12] K. Saalwächter, Progr. NMR Spectrosc. 51, 1 (2007).

[13] K. Kremer and G. S. Grest, J. Chem. Phys. 92, 5057 (1990).

[14] M. Kröger and S. Hess, Phys. Rev. Lett. 85, 1128 (2000).

[15] P. V. K. Pant and D. N. Theodorou, Macromolecules 28, 7224 (1995).

[16] R. Auhl, R. Everaers, G. S. Grest, K. Kremer, and S. J. Plimpton, J. Chem. Phys. 119, 12718 (2003).

[17] R. Everaers, S. K. Sukumaran, G. S. Grest, C. Svaneborg, A. Sivasubramanian, and K. Kremer, Science 303, 823 (2004).

[18] S. K. Sukumaran, G. S. Grest, K. Kremer, and R. Everaers, J. Polym. Sci, Part B: Polym. Phys. 43, 917 (2005).

[19] M. Kröger, Comp. Phys. Comm. 168, 209 (2005).

[20] S. Shanbhag and R. G. Larson, Phys. Rev. Let. 94, 
076001 (2005).

[21] Q. Zhou and R. G. Larson, Macromolecules 38, 5761 (2005).

[22] C. Tzoumanekas and D. N. Theodorou, Macromolecules 39, 4592 (2006).

[23] N. Uchida, G. S. Grest, and R. Everaers, J. Chem. Phys. 128, 044902 (2008).

[24] R. S. Hoy, K. Foteinopoulou, and M. Kröger, Phys. Rev. E 80, 031803 (2009).

[25] S. Plimpton, J. Comp. Phys. 117, 1 (1995).

[26] A. E. Likhtman, S. K. Sukumaran, and J. Ramirez, Macromolecules 40, 6748 (2007).

[27] R. Everaers and K. Kremer, Macromolecules 28, 7291 (1995).

[28] [1] Eqs.(7.137)-(7.141).

[29] M. Rubinstein and S. Panyukov, Macromolecules 35,
6670 (2002).

[30] P. E. Rouse, J. Chem. Phys. 21, 1273 (1953).

[31] A. Likhtman and T. McLeish, Macromolecules 35, 6332 (2002).

[32] J. des Cloizeaux, Europhys. Lett. 5, 437 (1988).

[33] D. J. Read, K. Jagannathan, and A. E. Likhtman, Macromolecules 41, 6843 (2008).

[34] E. van Ruymbeke, D. Vlassopoulos, M. Kapnistos, C. Y. Liu, and C. Bailly, Macromolecules 43, 525 (2010).

[35] M. Rubinstein and R. H. Colby, J. Chem. Phys. 89, 5291 (1988).

[36] Ref. 24] gives a value of $N_{e}=85$; we estimate the systematic error related to the use of different PPA algorithms and extraplation schemes to be of the order of \pm 7 . 